DOI: $10.1515 / \mathrm{adms}-2017-0018$

L. Mourokh ${ }^{1,2}$ P. Lazarev ${ }^{2}$

${ }^{1}$ Department of Physics, Queens College of CUNY, Flushing, NY 11367, USA and The

Graduate Center of CUNY, New York, NY 10016, USA, lev.murokh@qc.cuny.edu

${ }^{2}$ Capacitor Sciences Inc. 1605 Adams Drive, Suite B, Menlo Park, CA 94025

\title{
ENERGY STORAGE: DIELECTROPHORES - MOLECULES WITH NON-LINEAR POLARIZABILITY
}

\begin{abstract}
We examine the feasibility of film capacitors based on dielectrics with high non-linear polarizability as energy storage devices. Capacitors with increased energy density can be built by using composite materials with aromatic molecules (high polarizability) and envelope of alkyl tails (high resistivity). We determine the impact of the second order non-linearity onto energy density and translate high energy density requirements into molecular parameters necessary for high-performance capacitors. The relationship of permittivity and molecular polarizability is obtained by means of the non-linear Clausius-Mossotti equation. In order to demonstrate the the selection process for the molecular composition of dielectrophores, we compare several molecules, using quantum chemistry algorithms (Gaussian09). Starting from Langhals perylene (LP), we proceed with the nitrophenyl-perylene having one NH2 group (donor) and one NO2 group (acceptor). We show that, while their linear polarizabilities are comparable, the hyperpolarizabilities differ by several orders of the magnitudes. Two NH2 and NO2 groups can be attached to the nitro-naphthalene-perylene further increasing of the hyperpolarizability. Even larger polarization can be achieved by additional rylene groups increasing the polarizable electronic mass. We demonstrate that with such molecular engineering, capacitors can have the energy density which is attractive for practical applications.
\end{abstract}

Keywords: organic molecules, second-order permittivity, hyperpolarizability, stored energy density

\section{INTRODUCTION}

Energy storage is a large and fast growing market with applications in electrical vehicles, homes, industrial and commercial buildings, as well as in electronic devices. One of the most pressing industrial tasks is the amendment of intermittent energy sources, such as solar cells and wind turbines, by effective storage. To achieve such ambitious goal, a broad collaboration between various scientific communities is necessary. Only joint effort by the physicists underlying original principles of the operation, chemists developing advanced materials and structures, and electrical engineers constructing novel devices, would lead to ultimate success. As a part of this process, Capacitor Sciences Inc. develops dielectric composite materials for storage cells based on capacitors, where the energy is stored electrostatically [1].

In the present paper, we study the feasibility of conjugated molecules with large secondorder permittivity for applications in capacitors. We suggest using the layered structure to achieve both large polarization and large resistivity. Preliminary results lead us to believe that the proposed approach paves the way to new generation of capacitive energy storage. 
Conjugated molecules form molecular pi-stacks with uni-dimensional crystalline structure. Pi-stacks of conjugated molecules normally exhibit properties of semiconductors and might even be modified to make the transition to metallic conductivity [2]. We use the aromatic molecules as our fundamental units for the present model. Similar molecular materials in crystalline or colloidal form have demonstrated relatively high dielectric constants up to $2.0 \times 105$ (at $1 \mathrm{kHz}$ ) [3.4]. The polarizability in these systems shows a nonlinear dependence with respect to the electric field which makes such systems most suitable for energy storage purposes because its contribution is largest in static fields. Similar molecules were shown to exhibit strong non-linear optical properties [5] and, correspondingly, we can expect substantial polarizability in these structures. Recently, it was suggested to use organic molecules for high-capacitance dielectric materials [6,7]. The general donor-bridge-acceptor configuration was proposed with the account of non-linearity [6] and the response of well-known molecules were calculated [7], but no direct recommendation for the device geometry or specific paths to increase the capacitance were provided.

Aromatic conjugated core molecules are polarizable and in bulk structures are semiconductors or are even conductors. For energy storage applications the dielectric material must combine polarizability and resistivity to electronic transport. By providing nonconductive, resistive envelops to polarizable structures we can satisfy both requirements polarizability and resistivity. Aromatic molecules with high polarization are surrounded by aliphatic alkyl tails. Growth of similar structures was discussed in Refs. [8,9]. Such combinations can be approximated by microscopic molecular organization, with alternating layers of mostly polarizable cores and high resistive layers of tails. One possible arrangement is shown in Fig. 1.

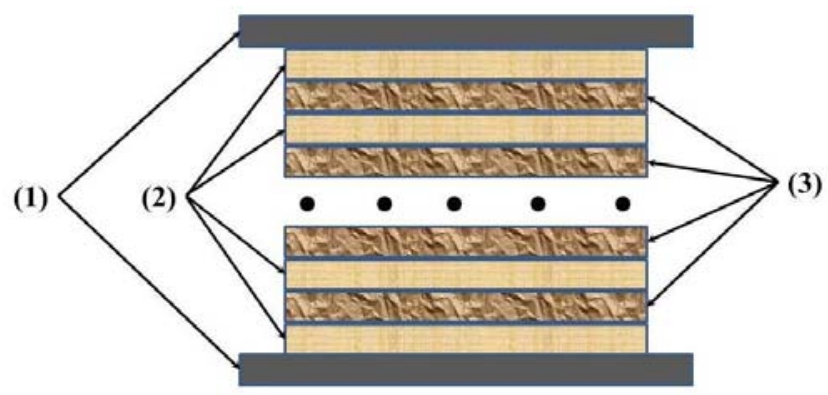

Fig. 1. Alternating layers of material: (1) metallic contacts, (2) high breakdown voltage materials, and (3) high dielectric permittivity layers

We study this system as a candidate for material structure that would satisfy both requirements for properties of the dielectric for the efficient energy storage.

The rest of the paper is structured as follows. In Section 2, we demonstrate the contributions of the second-order permittivity to the energy stored in the capacitor. We derive the relations between nonlinear permittivity and polarizability in Section 3 adopting the Clausius-Mossotti relation to the non-linear case. Several structures are examined and compared in Section 4 with their polarizabilities determined by Gaussian09 software [10]. Conclusions are presented in Section 5. 


\section{SECOND-ORDER PERMITTIVITY: CONTRIBUTIONS TO ENERGY DENSITY}

The energy density of an electric field is given by [11]

$w=\int_{0}^{D} \vec{E} \cdot d \overrightarrow{D^{\prime}}=\vec{E} \cdot \vec{D}-\int_{0}^{E} \vec{D} \cdot d \vec{E}^{\prime}$.

For simplicity, we consider the isotropic and uniform situation with all the fields directed along the $x$-axis. In this case, for nonlinear media the electric displacement can be written as

$D=\varepsilon_{0} \varepsilon^{(1)} E+\varepsilon_{0} \varepsilon^{(2)} E^{2}+\ldots$

Substituting Eq. (2) into Eq. (1), we obtain the following equation for energy density

$w=\varepsilon_{0} \varepsilon^{(1)} \frac{E^{2}}{2}+\varepsilon_{0} \varepsilon^{(2)} \frac{2 E^{3}}{3}+\ldots$

For a flat-plate capacitor with plate area $A$, plate separation $d$, applied voltage $V_{o p}=E d$, and linear capacitance without dielectric $C_{0}=\varepsilon_{0} A / d$, the total stored energy is

$W=\int w d \mathrm{Vol}=\varepsilon_{e f f} C_{0} \frac{V_{o p}^{2}}{2}$

where the effective dielectric function is given by

$\varepsilon_{\text {eff }}=\varepsilon^{(1)}+\varepsilon^{(2)} \frac{4 V_{o p}}{3 d}+\ldots$

Therefore, one can see that a dielectric with large second-order permittivity can be used for heavy-duty capacitors especially if they are charged at high voltages.

To compare the predicted energy density with existing commercial products, it is convenient to express the energy density in Watt-hours/kilogram units, as

$$
\frac{W}{M}\left(\frac{W h}{k g}\right)=\frac{1}{3600} \frac{\varepsilon_{0}}{\rho} \frac{V_{o p}^{2}}{2 d^{2}} \varepsilon_{e f f},
$$

where $\rho$ is the mass density.

If we aim to the energy density of $1000 \mathrm{Wh} / \mathrm{kg}$, and operational voltage in the range 0.1 $0.5 \mathrm{~V} / \mathrm{nm}$, then $\varepsilon_{\text {eff }}$ should exceed 20,000. In this paper, we discuss organic molecules that exhibit high dielectric permittivity achieved by the contribution of the non-linear effect. 


\section{PERMITTIVITIES AND POLARIZABILITIES: LINEAR AND NONLINEAR CLAUSIUS- MOSSOTTI RELATIONS}

Linear and nonlinear permittivities can be estimated by using the generalization of the Clausius-Mossotti relation in terms of polarizability and hyperpolarizability (second-order polarizability). Macroscopic polarization is given by

$$
P=N p_{\text {ind }},
$$

where $p_{\text {ind }}$ is the induced dipole and $N$ is the density of the dipoles. On the other hand,

$$
P=D-\varepsilon_{0} E=\varepsilon_{0}\left(\varepsilon^{(1)}-1\right) E+\varepsilon_{0} \varepsilon^{(2)} E^{2}+\ldots
$$

The induced dipole can be expressed in terms of the local field

$$
E_{l o c}=E+\frac{P}{3 \varepsilon_{0}},
$$

as

$$
p_{\text {ind }}=\alpha E_{l o c}+\beta E_{l o c}^{2}+\ldots,
$$

where $\alpha\left(=\alpha_{x x}\right)$ and $\beta\left(=\beta_{x x x}\right)$ are the polarizability and hyperpolarizability, respectively. Substituting Eqs. (9) and (10) into Eq. (7) and comparing to Eq. (8), we can equalize the coefficients with the same power of the electric field $E$.

The linear term would give as the usual Clausius-Mossotti relation [12,13],

$$
\frac{\left(\varepsilon^{(1)}-1\right)}{\left(\varepsilon^{(1)}+2\right)}=\frac{N \alpha}{3 \varepsilon_{0}},
$$

whereas the quadratic term would provide the expression for the second-order permittivity, as

$$
\varepsilon^{(2)}=\frac{N \beta}{3 \varepsilon_{0}}\left(\varepsilon^{(1)}+2\right)\left(1+\frac{2}{3}\left(\varepsilon^{(1)}-1\right)+\frac{1}{9}\left(\varepsilon^{(1)}-1\right)^{2}\right) .
$$

Therefore, having values for $\alpha$ and $\beta$ it is possible to determine the first- and secondorder permittivities and, correspondingly, the energy stored in the nonlinear capacitor. However, at high density of conjugated organic molecules $\left(3 \times 10^{27} \mathrm{~m}^{-3}\right)[5]$ and high linear polarizability which we expect from our structures, the linear Clausius-Mossotti expression breaks down due to the so-called polarization catastrophe [14]. It can be seen from Eq. (11) that the formal solution for $\varepsilon^{(1)}$ becomes negative when the right side exceeds 1 . Accordingly, to estimate the parameters of the molecules needed to achieve large enough effective dielectric constants, we use $\varepsilon^{(1)}$ as a parameter and plot $\varepsilon_{\text {eff }}$ as a function of $\varepsilon^{(1)}$ and $\beta$ (in 
atomic units, 1 a.u. $=e^{3} a_{0}^{3} / E_{h}^{2}$ for $\beta$, where $e$ is the electron charge, $a_{0}$ is the Bohr radius, and $E_{h}$ is the Hartree energy) in Fig. 2 for $N=3 \times 10^{27} \mathrm{~m}^{-3}$ and $V_{o p} / d=1 \mathrm{~V} / \mathrm{nm}$.

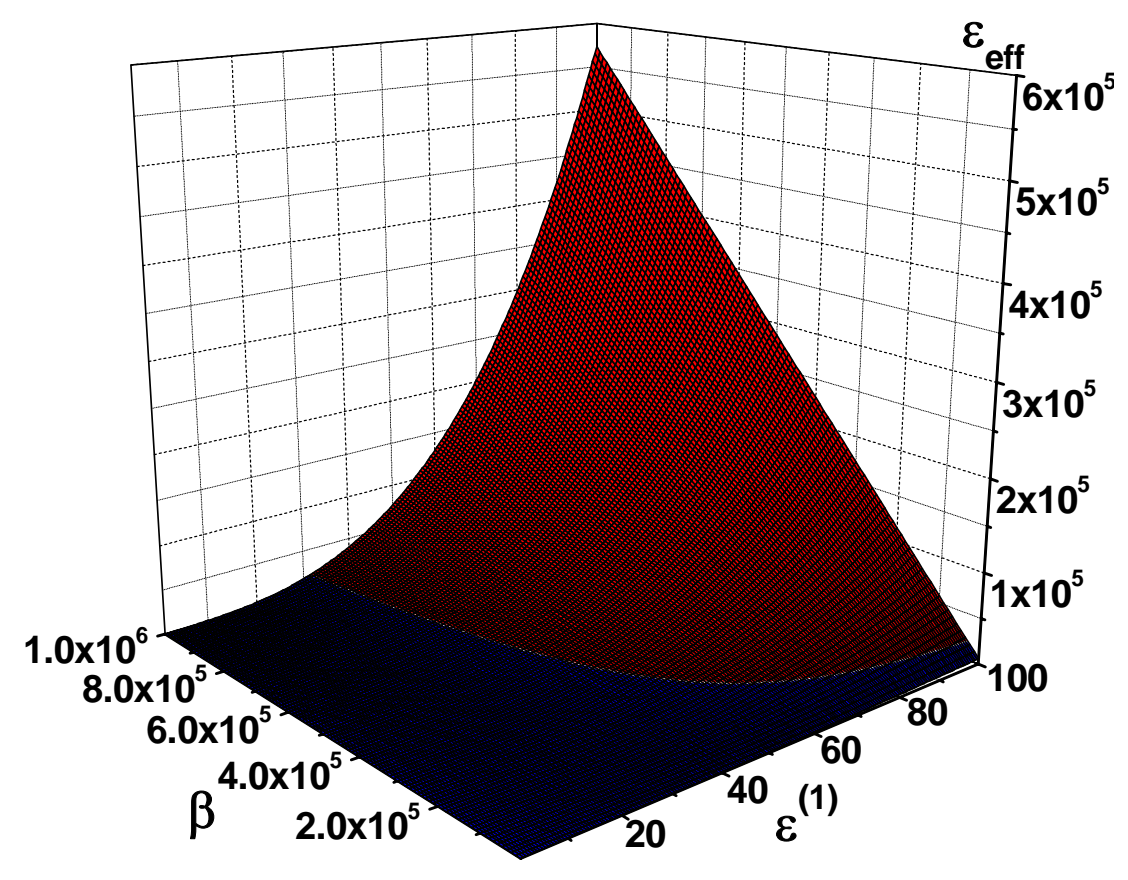

Fig. 2. Dependence of the effective dielectric constant on the first-order permittivity and hyperpolarizability. The red region indicates the values of the parameters where the effective dielectric constant exceeds 20,000

One can achieve quite large effective dielectric constant values even for moderate $\varepsilon^{(1)}$ if the hyperpolarizability is large enough. The range of parameters when the effective dielectric constant exceeds the desirable value of 20,000 is shown in red. In particular, for $\varepsilon^{(1)}=50, \beta$ should be close to a half of million.

\section{RESULTS}

We examine four specific molecules, Langhals perylene (LP), Fig. 3(a), nitro-phenylperylene with one $\mathrm{NO}_{2}$ group and one $\mathrm{NH}_{2}$ group (NPP), Fig. 3(b), nitro-naphthaleneperylene with two $\mathrm{NO}_{2}$ groups and two $\mathrm{NH}_{2}$ groups (NNP), Fig. 3(c), and NNP structure with additional rylene groups in the middle (NPPL), Fig. 3(d). Phenyl rings are added at the edges as linkers to the alkyl tails. The $\mathrm{NH}_{2}$ groups serving as electron donors and the $\mathrm{NO}_{2}$ groups serving as electron acceptors would create large polarizabilities and hyperpolarizabilities. They can be even be increased with enhanced charge separation in the case of NPPL.

These molecules are analyzed with Gaussian09 software [10], their polarizabilities and hyperpolarizabilities are determined using the B3LYP method with 6-31G+ basic sets and are given in Table 1. They are expressed in atomic units, 1 a.u $=e^{2} a_{0}^{2} / E_{h}$ for $\alpha$ and 1 a.u. 
$=e^{3} a_{0}^{3} / E_{h}^{2}$ for $\beta$, where $e$ is the electron charge, $a_{0}$ is the Bohr radius, and $E_{h}$ is the Hartree energy.
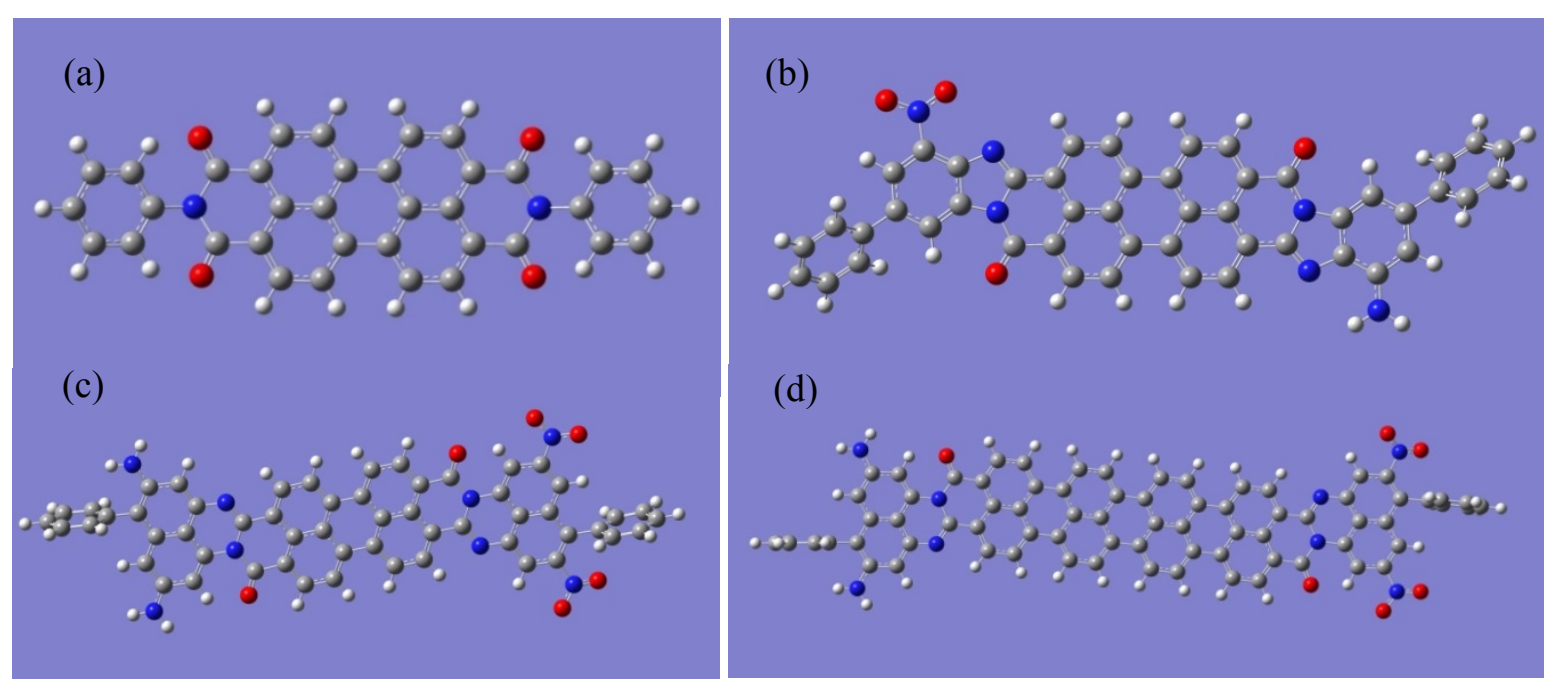

(d)

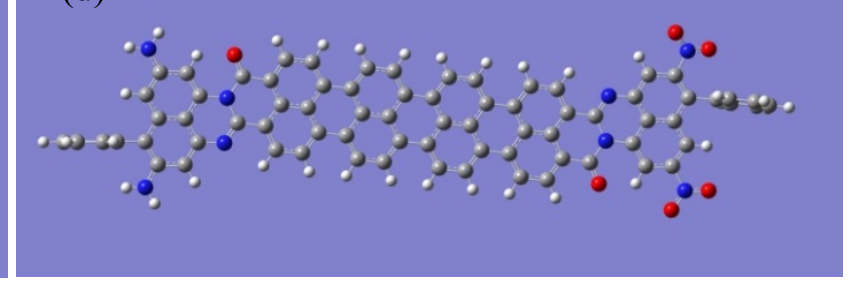

Fig. 3. (a) Langhals perylene. (b) Nitro-phenyl -perylene (NPP) with one NO2 and one NH2 groups. (c) Nitronaphthalene-perylene (NNP) with two NO2 and NH2 groups each. (d) NNP molecule with additional rylene groups in the middle (NNPL). Carbon, hydrogen, nitrogen, and oxygen atoms are shown by grey, white, blue, and red colors, respectively

Table 1. Polarizabilities and hyperpolarizablities for molecules of Fig. 3

\begin{tabular}{|c|c|c|c|c|}
\hline & LP & NPP & NNP & NNPL \\
\hline$\alpha$ & 945 & 1682 & 2057 & 3766 \\
\hline$\beta$ & 0.041 & 74,595 & 168,081 & 471,188 \\
\hline
\end{tabular}

One can see that while the linear polarizabilities for these molecules are comparable, the inclusion of the donor and acceptor groups leads to the increase of the hyperpolarizabilities by several orders of magnitude. Doubling the donor/acceptor groups increases the hyperpolarizability by more than three times, and additional rylene groups almost triple it again. The value of $\beta$ for the NNPL molecule is close to a half of million, so this molecule can be an elementary unit for the organic capacitor having its energy density comparable or exceeding that of the current generation of lithium-ion batteries.

\section{CONCLUSIONS}

Present paper demonstrates the feasibility of composite materials comprising non-linear polarizable cores - named dielectrophores - enveloped within resistive alkyl chains for applications in film capacitors suitable for practical applications. These molecules should contain aromatic cores having high polarizability and hyperpolarizability and alkyl tails with 
high dielectric strength. We have emphasized the role of nonlinear polarization and established the relationship between non-linear permittivity and the molecular hyperpolarizability. We have determined the material parameters for which the electrostatic capacitors based on proposed supramolecules are comparable or even exceed the characteristics of present-day energy storage devices. Using quantum chemistry modeling, we have studied a series of perylene-based molecules demonstrating that the introduction of donor and acceptor groups to the structure drastically increases its hyperpolarizability. This result is expected based on literature and results produced in non-linear optics studies [15]. Additional increase of polarizability can be achieved by increasing of the molecular length, i.e. by increasing of amount of electrons available for displacement along the molecular structure. According to initial results of the model, for a sufficiently long molecule with two donor and acceptor groups each, the hyperpolarizability reaches almost 500,000 which is sufficient for capacitive energy density comparable to that of lithium ion batteries. In summary, in our paper, we have (i) suggested to use novel physical principle, nonlinear polarizability; (ii) recommended novel type of materials, perylene-based conjugated polymers; and (iii) proposed novel engineering solution: layered structure to achieve both high polarization and high resistance.

\section{ACKNOWLEDGEMENT}

Authors are thankful to Prof. Seth Marder for valuable discussions and, in particular, for the suggestion of the term "dielectrophore".

\section{REFERENCES}

1. Hao X., A review on the dielectric materials for high energy-storage application, J. Adv. Dielect. 3, 1330001 (2013).

2. Shirakawa H., Louis E. J., MacDiarmid A. G., Chiang C. K., Heeger A. J., Synthesis of electrically conducting organic polymers: Halogen derivatives of polyacetylene, $(\mathrm{CH}) \mathrm{x}, \mathrm{J}$. Chem. Soc., Chem. Comm. 16, 578 (1977).

3. Hartman R. D. Pohl H. A., Hyperelectronic polarization in macromolecular solids, J. Polymer Sci. Part A-1: Polymer Chem. 6, 1135 (1968).

4. Ho C.-H., Liu C.-D., Hsieh C.-H., Hsieh K.-H., Lee S.-N., High dielectric constant polyaniline/poly(acrylic acid) composites prepared by in situ polymerization, Synth. Met. 158, 630 (2008).

5. Bosshard Ch., et al, Organic Nonlinear Optical Materials (Cordon and Breach Publishers, 1995).

6. Heitzer H. M., Marks T. J., Ratner M. A., Molecular donor-bridge-acceptor strategies for highcapacitance organic dielectric materials, J. Am. Chem. Soc. 137, 7189 (2015).

7. Heitzer H. M., Marks T. J., Ratner M. A, Computation of dielectric response in molecular solids for high capacitance organic dielectrics, Acc. Chem. Res. 49, 1614 (2016).

8. Kim H., Park J.-W., Self-assembly of rod-coils consisting of tetraaniline and alkyl chains in different oxidation states, J. Mater. Chem. 20, 1186 (2010). 
9. Shao Z., Yu Z., Hu J., Chandrasekaran S., Lindsay D. M., Wei Z., Faul C. F. J., Block-like electroactive oligo(aniline)s: anisotropic structures with anisotropic function, J. Mater. Chem. 22, 16230 (2012).

10. Gaussian 09, Revision E.01, M. J. Frisch, et al, Gaussian, Inc., Wallingford CT, 2009.

11. Landau L.D., Lifshitz E.M., Pitaevskii L.P., Electrodynamics of Continuous Media. Vol. 8, 2nd ed., (Butterworth-Heinemann, 1984).

12. Mossotti O. F., Discussione analitica sull'influenza che l'azione di un mezzo dielettrico ha sulla distribuzione dell'elettricità alla superficie di più corpi elettrici disseminati in esso, Memorie di Mathematica e di Fisica della Società Italiana della Scienza Residente in Modena 24, 49 (1850).

13. Clausius R., Abhandlungen über die mechanische Wärmetheorie, vol. 2 (Friedrich Vieweg und Sohn, Braunschweig, 1867).

14. Kittel Ch., Introduction to Solid State Physics, $8^{\text {th }}$ ed. (John Wiley \& Sons, 2005).

15. Liu X., Yang Z., Wang D., Cao H., Molecular structures and second-order nonlinear optical properties of ionic organic crystal materials, Crystals 6, 158 (2016). 